\title{
Molecular dynamics simulation of hydrogen enhancing dislocation emission
}

\author{
ZHOU Guohui (周国辉) ${ }^{1}$, ZHOU Fuxin (周富信) ${ }^{2}$, ZHAO Xuedan (赵雪丹) ${ }^{3}$, \\ ZHAN G Wenqing (张文清) ${ }^{3}$, CHEN Nanxian (陈难先) ${ }^{3}$, WAN Farong (万发荣) ${ }^{1}$ \\ and CHU Wuyang (褚武扬) ${ }^{1}$
}

(1. Department of Material Physics, University of Science and Technology Beijing, Beijing 100083, China;

2. Institute of Mechanics, Chinese Academy of Sciences, Beijing 100080, China;

3. Department of Physics, University of Science and Technology Beijing, Beijing 100083, China)

Received J uly 29, 1997

\begin{abstract}
The interactive pair potential between $\mathrm{Al}$ and $\mathrm{H}$ is obtained based on the ab initio calculation and the Chen-Möbius 3D lattice inversion formula. By utilizing the pair potentials calculated, the effects of hydrogen on the dislocation emission from crack tip have been studied. The simulated result shows that hydrogen can reduce the cohesive strength for $\mathrm{Al}$ single crystal, and then the critical stress intensity factor for partial dislocation emission decreases from $0.11 \mathrm{MPa} \sqrt{\mathrm{m}}\left(C_{\mathrm{H}}=0\right)$ to $0.075 \mathrm{MPa} \sqrt{\mathrm{m}}\left(C_{\mathrm{H}}=0.72 \%\right)$ and $0.06 \mathrm{MPa} \sqrt{\mathrm{m}}\left(C_{\mathrm{H}}=1.44 \%\right)$. This indicates that hydrogen can enhance the dislocation emission. The simulation also shows that atoms of hydrogen can gather and turn into small bubbles, resulting in enhancement of the equilibrium vacancy concentration.
\end{abstract}

Key words : hydrogen, dislocation emission, molecular dynamics, computer simulation.

Many experiments show that hydrogen can enhance dislocation emission, multiplication and motion ${ }^{[1,2]}$. In order to process the molecular dynamics simulation of hydrogen enhancing dislocation emission, we need not only the potentials of pure metal and hydrogen, but also the interatomic potential between the metal and hydrogen atoms. The key problem is how to obtain the interaction potential between the metal and hydrogen atoms. Generally, the interatomic potential can be obtained from the cohesive energy $E(x)$ which can be given by first principle calculation or experimental results of some simply ordered crystals. In this paper, we present a method to get the effective interactive potential between the metal and non metal atoms from crystals with simple virtual structure. The cohesive energy curves of the assumed virtual structure, which is composed of metal and non-metal atoms, are calculated by the self-consistent total energy linearized augmented plane wave (LAPW) method ${ }^{[3]}$. By using the Chen-Möbius 3D lattice inversion formula $^{[4]}$, the interactive potential between the metal and non metal atoms is then obtained.

After the potentials of $\mathrm{Al}-\mathrm{Al}, \mathrm{H}-\mathrm{H}$ and $\mathrm{Al}-\mathrm{H}$ are obtained, we use them to investigate how hydrogen influences dislocation emission in Al crystal. According to the critical stress intensity factor for the first partial dislocation emission for $\mathrm{Al}$ crystal containing varied hydrogen concentrations, we can judge the influence of hydrogen.

\section{The interaction potential bet ween $\mathrm{Al}$ and $\mathrm{H}$}

As we know, the binding energy $E(x)$ of the metal and alloy can be represented by the Rose equation of state ${ }^{[5]}$ : 


$$
\begin{aligned}
& E(x)=-E_{0}\left(1+a^{*}\right) \exp \left(-a^{*}\right), \\
& a^{*}=\sqrt{\frac{9 B \Omega}{E_{0}}}\left(\frac{x}{a_{0}}-1\right) .
\end{aligned}
$$

Here $B$ is the bulk modulus, $x$ is the current lattice constant, $E_{0}$ is the equilibrium binding energy and $\Omega$ is the equilibrium volume per atom. The parameters $B, E_{0}$ and $a_{0}$ in eq. (1) are calculated with the self-consistent total energy LAPW method. The calculated data for Al, solid H (fcc structure) and the $\mathrm{AlH}$ alloy (B2 structure) are listed in table 1.

Table $1 \quad B, E_{0}$ and $a_{0}$ from the first principle calculation

\begin{tabular}{cccc}
\hline & $B / 10^{11} \mathrm{~N} \cdot \mathrm{m}^{-2}$ & $E_{0} / 1.60 \times 10^{-19} \mathrm{~J}$ & $a_{0} / 10^{-10} \mathrm{~m}$ \\
\hline $\mathrm{Al}$ & 0.99 & 3.34 & 4.03 \\
$\mathrm{H}$ & 1.21 & 1.36 & 2.27 \\
$\mathrm{AlH}$ & 0.75 & 2.03 & 2.07 \\
\hline
\end{tabular}

Based on the data listed in table 1, the cohesive energies for $\mathrm{Al}, \mathrm{H}$ and $\mathrm{AlH}$, i.e. $E_{\mathrm{Al}}, E_{\mathrm{H}}$ and $E_{\mathrm{AlH}}$, can be obtained according to equation (1).

The binding energy can be written in the sum of the air potential between two atoms, i.e.

$$
E(x)=\frac{1}{2} \sum_{1 \rightarrow 0} \Phi(\mathrm{R})=\frac{1}{2} \sum_{n} r_{n} \Phi\left(\mathrm{a}_{n} x\right),
$$

where $r_{n}$ is the coordination number, $\boldsymbol{a}_{n}$ is the ratio between the distance of the $n$th shell and the NN distance $x$. Based on the Chen-Möbius 3D lattice inversion method, the pair potential $\Phi(x)$ can be converted. The formula for converting the pair potential $\Phi(x)$ from the cohesive energy $E(x)$ can be read as

$$
\Phi(x)=\sum_{n} \mu\left(\mathbf{a}_{n}\right) E\left(\mathbf{a}_{n} x\right) .
$$

Here $\mu\left(\mathbf{a}_{n}\right)$ is the inversion coefficient. Readers who show interest in this formula can find the detailed explanation of these parameters in ref. [4]. From eq. (3) the potentials $\Phi_{\mathrm{Al}}(x)$ and $\Phi_{\mathrm{H}}(x)$ can be obtained.

We can treat the cohesive energy $E_{\mathrm{AlH}}$ as the sum of the cohesive energy $E_{\mathrm{Al}}{ }^{*}, E_{\mathrm{H}}{ }^{*}$ and the interaction energy $E_{\mathrm{Al}-\mathrm{H}}$, i.e.

$$
E_{\mathrm{AlH}}=E_{\mathrm{Al}}{ }^{*}+E_{\mathrm{H}}{ }^{*}+E_{\mathrm{ArH}} \text {. }
$$

Here $\mathrm{Al}$ and $\mathrm{H}$ are the simple cubic structures, and the alloy $\mathrm{AlH}$ is the $\mathrm{B} 2$ structure which consists of two simple cubic structures. We assume that these potentials are transferable from fcc to $\mathrm{SC}$ or $\mathrm{B} 2$ structures. In this case, $E_{\mathrm{Al}}{ }^{*}$ and $E_{\mathrm{H}}{ }^{*}$ can be expressed as

$$
\begin{aligned}
E_{\mathrm{Al}}{ }^{*}(x) & =\frac{1}{2} Y_{\mathrm{Al}} \sum_{\mathrm{R}} \Phi_{\mathrm{Al}} \Phi_{\mathrm{Al}}(\mathrm{R}), \\
E_{\mathrm{H}}{ }^{*}(x) & =\frac{1}{2} Y_{\mathrm{H}} \sum_{\mathrm{R}} \Phi_{\mathrm{H}} \Phi_{\mathrm{H}}(\mathrm{R}),
\end{aligned}
$$

where $Y_{\mathrm{Al}}$ and $Y_{\mathrm{H}}$ are atomic ratios of $\mathrm{Al}$ and $\mathrm{H}$ in the alloy $\mathrm{AlH}$. From eqs. (4) - (6), we have

$$
E_{\mathrm{Al}-\mathrm{H}}(x)=E_{\mathrm{AlH}}-\frac{1}{2} Y_{\mathrm{Al}} \sum_{\mathrm{R}_{\mathrm{Al}} \neq 0} \Phi_{\mathrm{Al}}(\mathrm{R})-\frac{1}{2} Y_{\mathrm{H}} \sum_{\mathrm{R}} \Phi_{\mathrm{H}} \Phi_{\mathrm{H}}(\mathrm{R}) \text {. }
$$

Substituting the data of $\mathrm{AlH}$ listed in table 1 into eq. (1), we can get the first item of the right- 


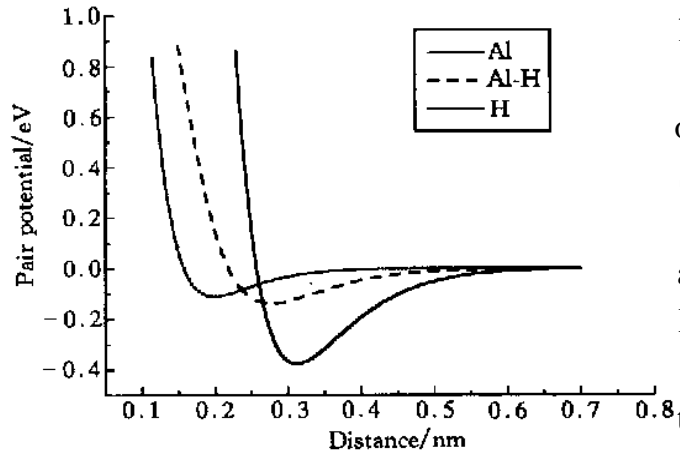

Fig. 1. The pair potentials for $\mathrm{Al}, \mathrm{H}$ and $\mathrm{Al}-\mathrm{H}$ as a function of distance of separation. hand side of eq. (7), i. e. $E_{\mathrm{AlH}} . \Phi_{\mathrm{Al}}(x)$ and $\Phi_{\mathrm{H}}$ $(x)$ can be obtained based on eq. (3), and the second and third items in eq. (7) can be calculated. Substituting $E_{\mathrm{Ar}-\mathrm{H}}$ obtained from eq. (7) into eq. (3), the interactive potential between $\mathrm{Al}$ and $\mathrm{H}$ atoms $\Phi_{\mathrm{ArH}}(x)$ can be inverted using the ChenMöbius 3D lattice inversion method.

From the procedures we can obtain the pair potentials for $\mathrm{Al}-\mathrm{Al}, \mathrm{Al}-\mathrm{H}$ and $\mathrm{H}-\mathrm{H}$. The pair potential curves $\Phi_{\mathrm{Al}}(x), \Phi_{\mathrm{H}}(x)$ and $\Phi_{\mathrm{Al}-\mathrm{H}}(x)$ are shown in fig. 1. The interaction force $F=\frac{\partial \Phi}{\partial X}$ between $\mathrm{Al}-\mathrm{Al}$ and $\mathrm{Al}-\mathrm{H}$ atoms can be calculated based on the pair potential curves.

\section{Calculation method of molecular dynamics simulation}

The border discrete atoms are prescribed by the mode II anisotropic elastic $K$ displacement field. The loading rate $\dot{K}_{\mathrm{II}}$ is used as a loading parameter. In the present simulation, $\dot{K}_{\mathrm{II}}=$ $0.02 \mathrm{MPa} \cdot \sqrt{\mathrm{m}} / \mathrm{ps}$. The inner atoms follow law of Newton

$$
\mathrm{F}_{i}=-\frac{\partial E_{\mathrm{tot}}}{\partial \mathrm{r}_{i}}=m_{i} \dot{\mathrm{v}_{\mathrm{i}}}
$$

The present molecular dynamics calculations are carried out by the Leapfrog Algorithm ${ }^{[6]}$. In eq. (8) $m_{i}$ is the mass of the $i$ th atom. The time step in the present calculation is taken to be $10^{-3}$ ps. Because the hydrogen atom moves much faster than the $\mathrm{Al}$ atom, the time step chosen is very small in order to avoid collision between $\mathrm{Al}$ and $\mathrm{H}$ atoms. In all the simulations, the temperature of the systems is maintained at $40 \mathrm{~K}$. The initial velocity is the Maxwell-Boltzmann distribution corresponding to a given temperature.

The $\{110\},\{111\}$ and $\{112\}$ crystallographic planes of the parallelepiped with a slit are used in the present calculations. The coordinate system is selected to be $x, y$ and $z$ axes in $\langle 110\rangle,\langle 112\rangle$ and $\langle 111\rangle$ directions respectively. In the FCC crystal, a full dislocation moves in the $\langle 110\rangle$ direction in $\{111\}$ plane. So, in the present model, the crack plane is taken to be in the $\{111\}$ plane, and the crack front is in the $\langle 112\rangle$ direction. Hydrogen usually lies in the octahedral interstitial of the fcc crystal. In fig. 2, the solid circle represents $\mathrm{Al}$ atom, while" + " represents hydrogen atom. Under mode II loading, the dislocations move in the $\langle 110\rangle$ direction. The periodicity in $\langle 111\rangle \mathrm{di}^{-}$ rection is in three layers, in $\langle 110\rangle$ direction in two layers and in $\langle 112\rangle$ direction in six layers.

The boundary conditions applied to the

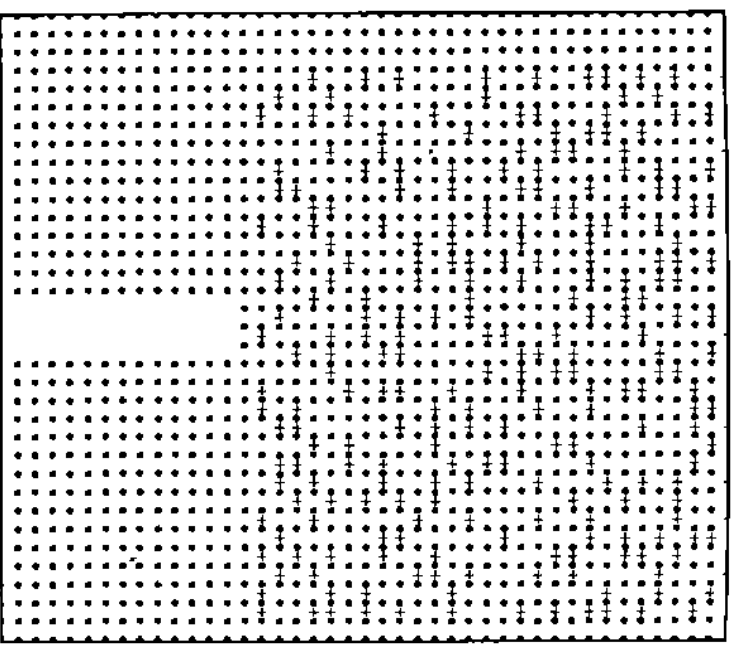

Fig. 2. The initial atomic configuration of hydrogen distributed randomly ahead of the crack tip. 
boundary of the discrete atom region in our molecular dynamics simulation have been that of a prescribed displacement distribution dictated by a mode II anisotropic $K$ field in the $x-z$ plane. In the $y$-direction, a six layer periodic representation is applied. Therefore the present atom lattice is actually three-dimensional. The total number of atoms for the simulation is $N=8340$. The length in the $x$-direction is $(\sqrt{2} / 4) \times 140 a_{0}\left(a_{0}\right.$ is the lattice constant $)$ and the width in the $z$-direction is $(\sqrt{3} / 3) \times 60 a_{0}$. The distance between the crack tip and the left boundary is $(\sqrt{2} / 4) \times$ $20 a_{0}$. As the length from the crack tip to the right boundary is large enough, the effect of the boundary constraints on the nucleation and emission of dislocations can be neglected if the dislocations are far from the boundary. Hydrogen atoms are placed randomly in the octahedral interstices of the region of $10 \sqrt{2} a_{0} \times 10 \sqrt{3} a_{0}$. The total number of interstices is 1200 . In the present simulations, the numbers of hydrogen atoms are 60,120 and 360 respectively. There is no hydrogen atom out of this region. The initial atomic configuration is shown in figure 2.

\section{Simulated results and discussion}

If there is no hydrogen atom in $\mathrm{Al}$ crystal, at the 5 350th time step , the first partial dislocation is emitted from the crack tip, as shown in fig. 3 (a). That is to say that the critical stress intensity factor for partial dislocation emission in $\mathrm{Al}$ crystal $K_{\text {IIe }}(\mathrm{Al})$ is $0.107 \mathrm{MPa} \cdot \sqrt{\mathrm{m}}$. If 60 hydrogen atoms are distributed randomly in the small region ahead of the crack tip, where the hydrogen concentration (atomic percentage) is $0.72 \%$, the first partial dislocation will be emitted at the 3 750th time step, as shown in fig. 3(b). In this case, the critical stress intensity factor for partial dislocation emission in $\mathrm{Al}$ crystal containing $60 \mathrm{H}$ atoms decreases to $0.075 \mathrm{MPa} \cdot \sqrt{\mathrm{m}}$. If 120 hydrogen atoms are added to the region, the first partial dislocation is emitted from the crack tip at the 3 000th time step, as shown in fig. 3 (c). The critical stress intensity factor for partial dislocation emission decreases to $0.06 \mathrm{MPa} \cdot \sqrt{\mathrm{m}}$ further. When the run reaches 20000 time steps, we keep the intensity stress factor $K_{\mathrm{II}}=0.4 \mathrm{MPa} \cdot \sqrt{\mathrm{m}}$ constant, and there exist four partial dislocations in the pure $\mathrm{Al}$ crystal, while five dislocations in the crystal containing 60 hydrogen atoms. The results indicate that hydrogen can decrease the critical stress intensity factor for the dislocation emission and enhance the dislocation emission.

Under the influence of stress, hydrogen atoms lying in the octahedral interstitials can move

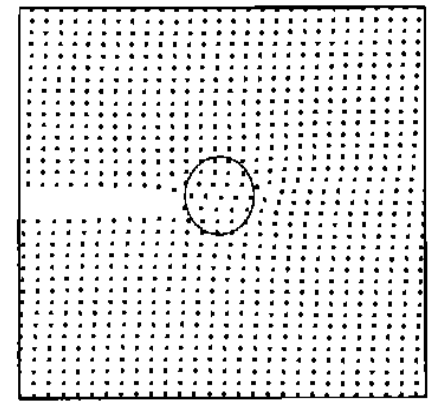

(a)

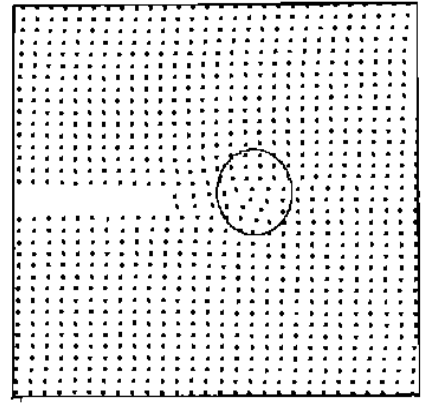

(b)

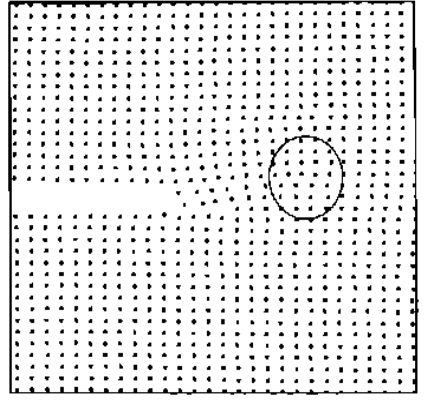

(c)

Fig. 3. (a) At the 5 350th time step, the first partial dislocation is emitted (no H atom). (b) At the 3750 th time step , the first partial dislocation is emitted (containing 60 hydrogen atoms). (c) At the 3000 th time step, the first partial dislocation is emitted (containing 120 hydrogen atoms). 


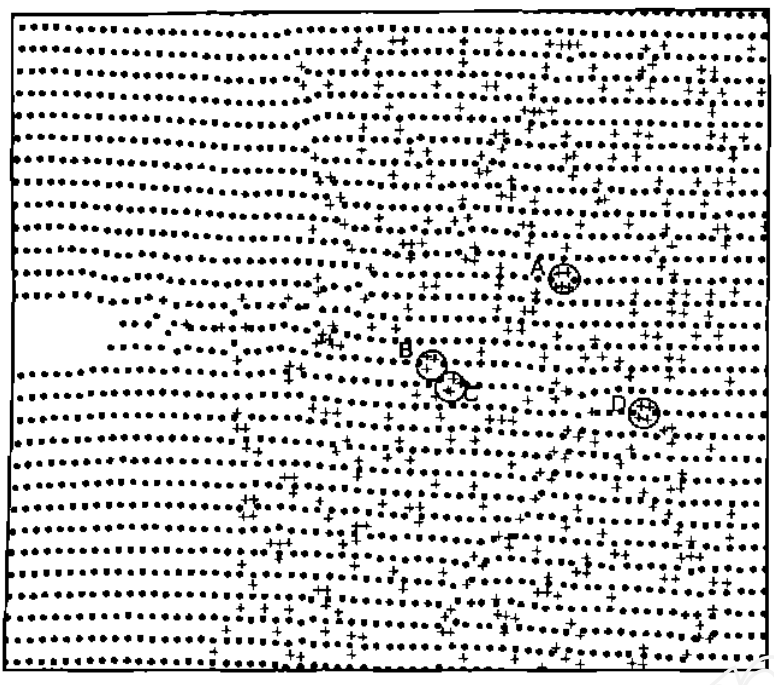

Fig. 4. At the 20000 th time step, some vacancies are zener.tte ahead of the crack tip.

and gather, resulting in generation of small voids or bubbles (including 3 to 5 hydrogen atoms), as shown by A, B , C and D in fig. 4. There are $360 \mathrm{H}$ atoms in the region. $\mathrm{H}$ atom is represented by the symbol" + ", and the time step is the $20000 \mathrm{th}$.

The reason that hydrogen enhances dislocation emission can be explained as follows. For a mode II crack, the critical stress intensity factor for the dislocation emission from the crack tip is read as ${ }^{[7]}$

$$
K_{\text {IIe }}=\left(\pi r_{0}\right)^{1 / 2}\left[\boldsymbol{\mu} b / 4 \pi(1-v) r_{0}+\mathrm{T}_{\mathrm{t}}\right] \text {. }
$$

Here $r_{0}=d / e$ is the dislocation core $\mathrm{ra}^{-}$ $\operatorname{dius}^{[8]}, d=\sqrt{6} / 3 a_{0}=3.31 \times 10^{-10} \mathrm{~m}$ is the effective plane distance, $\mu$ is the shear modulus, $\mathbf{T}_{\mathrm{f}}$ is the lattice resistance force, $b$ is

the Burgers vector. Substituting these parameters $b=\sqrt{6} / 6 a_{0}=1.65 \times 10^{-10} \mathrm{~m}, v=0.347, \mathrm{~T}_{\mathrm{f}}=$ $20 \mathrm{MPa}, \mu=26.3 \times 10^{3} \mathrm{MPa}$ into eq. (9), we get $K_{\text {IIe }}(\mathrm{Al})=0.12 \mathrm{MPa} \cdot \sqrt{\mathrm{m}}$.

Because the elastic modulus is relative to the cohesive strength $\sigma_{\text {th }}$, e.g. ref. [9],

$$
\sigma_{\text {th }}=E a_{0} / \Pi d=2 \mu(1+v) a_{0} / \Pi d .
$$

Here $d$ is the plane distance. Assume that hydrogen decreases only the cohesive strength $\sigma_{\text {th }}$ but does not affect $v, a_{0}$ and $d$. Based on eq. (10) we get

$$
\mu(\mathrm{H})=\left[\sigma_{\mathrm{th}}(\mathrm{H}) / \sigma_{\mathrm{th}}\right] \mu .
$$

Since the second item in eq. (9) is much less than the first item, and then can be neglected, from eqs. (9) and (11) we get

$$
K_{\text {IIe }}(H) / K_{\text {IIe }}(\mathrm{Al})=\sigma_{\text {th }}(\mathrm{H}) / \sigma_{\text {th }} .
$$

According to the difference of ion escaping work between the specimens which is charged and not charged with hydrogen, if we think that the cohesive energy equals the sum of the sublimation and ionization energy, then from the experimental result, we know that hydrogen with the atomic ratio 1 atm $\%\left(C_{\mathrm{H}}=0.01\right)$ can decrease the atomic cohesive strength by $5 \%^{[10]}$, i.e. when $C_{\mathrm{H}}$ $=0.01, \sigma_{\mathrm{th}}(\mathrm{H}) / \sigma_{\mathrm{th}}=0.95$. In the rectangle of fig. 2 there are 120 hydrogen atoms, and $C_{\mathrm{H}}$ $=0.1$, which will make the cohesive strength decrease by $50 \%$, that is to say, $\sigma_{\mathrm{th}}(\mathrm{H}) / \sigma_{\mathrm{th}}=$ 0.5. Molecular dynamics simulation shows that the first dislocation is emitted at the 5350 th time step for pure $\mathrm{Al}$ crystal, corresponding to $K_{\mathrm{IIe}}(\mathrm{Al})=0.11 \mathrm{MPa} \cdot \sqrt{\mathrm{m}}$; and at the 3000 th time step for the $\mathrm{Al}$ crystal containing $1.44 \%$ hydrogen atoms in the small region ahead of the crack tip , corresponding to $K_{\text {IIe }}(\mathrm{H})=0.06 \mathrm{MPa} \cdot \sqrt{\mathrm{m}}$. The ratio of the critical stress intensity factors is $K_{\text {IIe }}(\mathrm{H}) / K_{\text {IIe }}(\mathrm{Al})=0.55$, which conforms well with the result calculated based on eq. (12). It also shows that the pair potentials chosen are self-consistent with the results calculated.

Why does hydrogen cause the void density ahead of the loaded crack tip to increase ? Simirov et al. indicated that implantation action of hydrogen atoms will cause the void density to increase 
from $C_{\mathrm{V}}(0)$ to $C_{\mathrm{V}}(\mathrm{H})$, i.e. ${ }^{[11]}$

$$
C_{\mathrm{V}}(\mathrm{H}) / C_{\mathrm{V}}(0)=\exp \left(f_{\mathrm{H}} C_{\mathrm{H}} / K T\right) /\left(1-C_{\mathrm{H}}\right) \text {, }
$$

where $C_{\mathrm{H}}$ is the hydrogen concentration, $f_{\mathrm{H}}$ is the formation energy of hydrogen. Substituting $f_{\mathrm{H}}$ $=1 \mathrm{eV}, K T=0.1 \mathrm{eV}$ and $C_{\mathrm{H}}=0.5$ into eq. (13), we get $C_{\mathrm{V}}(\mathrm{H}) / C_{\mathrm{V}}(0)=300$. It shows that the void density increases by two orders of magnitude.

\section{Conclusion}

The molecular dynamics simulation results show that:

1) Hydrogen makes the critical stress intensity factor for dislocation emission decrease from $0.11 \mathrm{MPa} \cdot \sqrt{\mathrm{m}}\left(C_{\mathrm{H}}=0\right)$ to $0.075 \mathrm{MPa} \cdot \sqrt{\mathrm{m}}\left(C_{\mathrm{H}}=0.72 \%\right)$ and $0.06 \mathrm{MPa} \cdot \sqrt{\mathrm{m}}\left(C_{\mathrm{H}}=\right.$ $1.44 \%)$, i.e. hydrogen can enhance dislocation emission.

2) The stress induces hydrogen enrichment. Hydrogen atoms ahead of a loaded crack tip can gather and cause the equilibrium vacancy concentration to increase.

\section{References}

1 Robertson, I. M. , Birnbaum, H. K. , An HEVM study of hydrogen effect on the deformation and fracture of nickel, Acta Metall., 1986, 34:353.

2 Chu, W. Y., Wang, Y. B., Hsiao, C. M., Research of HIC in an aluminum alloy, Corrosion, $1982,38: 561$.

3 Krakauer, H. , Posternak, M. , Freeman, A. J . , Linearized augmented plane-wave method for the electronic band structure of thin films, Phys. Rev., 1979, B19: 1706.

4 Chen, N. X. , Li, M. , Liu, S. J. , Phonon dispersions and elastic constants of $\mathrm{Ni}_{3} \mathrm{Al}$ and Möbius inversion, Phys. Lett. , 1994, A195: 135.

5 Guinea, F. , Rose. J. H. , Smith, J. R. et al., Scaling relations in the equation of state, thermal expansions and melting of metals, A ppl. Phys. Lett., 1984, 144(1): 53.

6 Heermann, D. W. , Computer Simulation Methods in Theoretic Physics, 2nd ed., Berlin: Springer-Verlag, 1990.

$7 \mathrm{Ohr}$, S. M. , An electron microscope study of crack tip deformation and its impact on the dislocation theory of fracture, Mater. Sci. Eng., 1985, $72: 1$.

8 Hirth, J. P., Theory of Dislocation, 2nd ed., New York: John Wiley and Sons Inc., 1982.

9 Cherepanov, G. P. , Mechanics of B rittle Fracture (eds. De Wit, R., Cooley, W. C. ), New York: Mc Graw-Hill Inter. Book Co. , 1979.

10 Hsiao, C. M., Chu, W. Y., Hydrogen Effect in Metals (eds. Bernstein, I. M. , Thompson, A. W.), New York: AIME, $1981,255$.

11 Gavriljuk, V. G. , Bugaev, V. N., Petrov, Yu. N. et al. , Hydrogen-induced equilibrium vacancies in FCC iron-base alloys, Scr. Metall. Mater., 1996, 34: 903. 